\title{
What value can Google search data add to existing syndromic surveillance systems?
}

\author{
Helen K. Green ${ }^{\star 1}$, Obaghe Edeghere', Alex Elliot', Ingemar Cox², ${ }^{1}$, Rachel McKendry² \\ and Gillian Smith ${ }^{1}$
}

'Public Health England, Birmingham, United Kingdom; ${ }^{2}$ University College London, London, United Kingdom; ${ }^{3}$ University of

Copenhagen, Copenhagen, Denmark

\section{Objective}

To carry out an observational study to explore what added value Google search data can provide to existing routine syndromic surveillance systems in England for a range of conditions of public health importance and summarise lessons learned for other countries.

\section{Introduction}

Globally, there have been various studies assessing trends in Google search terms in the context of public health surveillance ${ }^{1}$. However, there has been a predominant focus on individual health outcomes such as influenza, with limited evidence on the added value and practical impact on public health action for a range of diseases and conditions routinely monitored by existing surveillance programmes. A proposed advantage is improved timeliness relative to established surveillance systems. However, these studies did not compare performance against other syndromic data sources, which are often monitored daily and already offer early warning over traditional surveillance methods. Google search data could also potentially contribute to assessing the wider population health impact of public health events by supporting estimation of the proportion of the population who are symptomatic but may not present to healthcare services.

\section{Methods}

We sought to determine the added public health utility of Google search data alongside established syndromic surveillance systems in England ${ }^{2}$ for a range of conditions of public health importance, including allergic rhinitis, scarlet fever, bronchitis, pertussis, measles, rotavirus and the health impact of heatwaves. Google search term selection was based on diagnostic and clinical codes underlying the syndromic indicators, with Google Trends ${ }^{3}$ used to identify additional related internet search terms. Daily data was extracted from syndromic surveillance systems ${ }^{2}$ and from the Google Health Trends Application Programming Interface (API) from 2012 to 2017 and a retrospective daily analysis undertaken during pre-identified public health events to identify a) whether signals were detected during these events and b) assess the correlation with analogous syndromic surveillance indicators through calculation of Spearman correlation coefficients and lag assessment to determine timeliness.

\section{Results}

We detected increases in Google search term frequency during public health events of interest. Good correlation was seen with comparable syndromic surveillance indicators on a daily timescale for several health outcomes, including the search terms hayfever, scarlet fever, bronchiolitis and heatstroke. Weaker correlation was seen for conditions which occur in small numbers and are vaccine preventable such as measles and pertussis. Lag analysis showed similar timeliness between daily syndromic and Google data, suggesting that, overall, Google data did not provide an earlier or delayed signal compared to syndromic surveillance indicators in England.

\section{Conclusions}

To the best of our knowledge this is the first time trends in Google search data have been compared against syndromic data for a range of public health conditions in England. These findings demonstrate the potential utility of internet search query data in conjunction with existing systems in England, with syndromic surveillance data found to be as timely as Google data. These findings also have important implications for countries where there are no such healthcarebased syndromic surveillance systems in place. Factors to consider with analyses of Google search trend data in the context of disease surveillance have been highlighted, including the choice of search terms and interpretation of the reasons behind searching the internet.

Keywords

Syndromic surveillance; Google; Public health

\section{Acknowledgments}

We acknowledge support from: NHS 111 and NHS Digital for their assistance and support in the NHS 111 system; $\mathrm{OOH}$ providers submitting data to the GPOOHSS and Advanced Heath \& Care; ED clinicians and NHS Trust staff supporting EDSSS, the Royal College of Emergency Medicine and technical support provided by EMIS Health and L2S2 Ltd; TPP and participating SystmOne practices and University of Nottingham, ClinRisk, EMIS and EMIS practices submitting data to the QSurveillance database and Google for access to their Google Health Trends API. The authors would like to thank Mr Paul Loveridge of the PHE Realtime Syndromic Surveillance Team (ReSST) and Dr Bill Lampos of the Department of Computer Science, UCL for their technical expertise. This work has been supported by the i-sense EPSRC IRC in Early Warning Sensing Systems for Infectious Diseases grant (EP/K031953/1) and a Google Research Sponsorship. HKG received support from i-sense to attend ISDS. RAM is supported by the Royal Society Wolfson Merit Award. AJE and GES are supported by the National Institute for Health Protection Research Health Protection Unit (NIHR HPRU) in Emergency Preparedness and Response at King's College London in partnership with PHE. The views expressed are those of the author(s) and not necessarily those of the NHS, the NIHR, the Department of Health or Public Health England.

\section{References}

${ }^{1}$ Nuti SV, Wayda B, Ranasinghe I, Wang S, Dreyer RP, Chen SI, Murugiah $\mathrm{K}$. The use of google trends in health care research: a systematic review. PLoS One. 2014 Oct 22;9(10):e109583.

${ }^{2}$ Public Health England. Syndromic surveillance: systems and analyses. 2017. Available online: https://www.gov.uk/government/collections/ syndromic-surveillance-systems-and-analyses ${ }^{3}$ Google. 2017. Google Trends. Available online: https://trends.google.com/trends/

\section{*Helen K. Green}

E-mail: helen.k.green@phe.gov.uk 